\title{
Applications of Three-Dimensional LBM-LES Combined Model for Pump Intakes
}

\author{
Miao Guo ${ }^{1}$, Xuelin Tang ${ }^{1,2, *}$, Yanwen $\mathrm{Su}^{1}$, Xiaoqin $\mathrm{Li}^{1,2}$ and \\ Fujun Wang ${ }^{1,2}$ \\ ${ }^{1}$ College of Water Resources and Civil Engineering, China Agricultural University, \\ Beijing 100083, China. \\ ${ }^{1}$ Beijing Engineering Research Centre of Safety and Energy Saving Technology for \\ Water Supply Network System, China Agricultural University, Beijing 100083, China. \\ Communicated by Kun Xu
}

Received 20 April 2017; Accepted (in revised version) 5 July 2017

\begin{abstract}
Lattice Boltzmann model (LBM) in conjunction with an accurate Large Eddy Simulation (LES) technology was proposed to simulate various vortical structures and their evolutions in open pump intakes. The strain rate tensor in the LES model is locally calculated by means of non-equilibrium moments based on Chapman-Enskog expansion, and bounce-back scheme was used for non-slip condition on solid walls and reflection scheme for free surface. The presented model was applied to investigate free-surface and wall-attached vortices for different water levels and flow rate. The vortex position, shapes and vorticities were predicted successfully under three flowing cases (i.e. critical water level (CWL), lower water level, lower flow rate), and the numerical velocity and streamline distribution were analyzed systematically. For CWL based on Froude number considering open channel flows, the shape and the location of various dynamic vortices were captured. Compare to the experimental results of CWL, more vortices were predicted for lower water level, and less vortices were observed for lower flow rate. The predicted velocities and vortex locations are in good agreement with the experimental of a small physical model. The comparisons demonstrated the feasibility and stability of above-mentioned model and numerical method in predicting vortex flows inside open pump intakes.
\end{abstract}

AMS subject classifications: 76F65, 76M25

Key words: Lattice Boltzmann model, Large Eddy Simulation, free-surface vortices, wall-attached vortices, open pump intakes.

*Corresponding author. Email addresses: GM10@cau.edu.cn (M. Guo), xl-tang@mail.tsinghua.edu.cn (X. Tang), ysu1452@163.com(Y. Su), 13522915056@163.com (X. Li), wangfj@cau. edu.cn (F. Wang) 


\section{Introduction}

The flows inside pump intakes are generally very complex and contains commonly many coherent structures. Poor designs of pump intakes and improper combinations of geometric parameters including pump bell give rise to bad flow pattern with various vortices, such as free surface vortices and wall-attached vortices [1], which influence safe and steady operation in pump stations, such as noise and vibration, impeller damage due to cavitation, and uneven impeller loadings [2-4].

Experimental techniques are effective and intuitive tools to investigate various vortices and corresponding generation causes and find practical solutions to eliminate or suppress them. Rajendran [5] used conventional flow visualization to identify the vortices, and obtained quantitative information on the number, location, shape, size, and strength of vortices by PIV technology in a simple but representative intake. Tomoyoshi et al. [6] studied the velocity and vorticity distribution in the sump using PIV method and visualization method, the experimental data show that the critical submergence for the free surface vortex and the wall-attached vortices are almost proportional to the flow rate in the intake. But experiments have some disadvantages such as high cost and long periods, and now numerical methods are useful and promising tools to investigate the flow field in pump intake and to optimize geometry parameters.

Tokyay et al. confirmed that LES model with a sufficiently fine mesh can accurately capture not only qualitatively but also quantitatively most of flow features in a pump intake [7]. Tang et al. [8] investigated the feasibility and applicability of turbulence models in predicting flows in the pump sump, and analyzed various vortex streamlines and strength in the sump. Aljaz Skerlavaj et al. [9] focused on the choice of a suitable turbulence model for flow simulations in an industrial pump intake, and successfully predicted the gas-core length of vortex for different submergence.

In recent years, emerging LBM becomes a popular tool to solve fluid flow problems. Tang et al. [10] used 2D LBM-SGS model to study the flow characteristics in a forebay, and compared with the experimental data, the numerical results show that the model scheme has the capacity to simulate complex flows in shallow water with reasonable accuracy and reliability. D. Bespalko et al. [11] simulated a turbulent channel flow by using the D3Q19 athermal LBM, and compared these numerical results to those calculated using Navier-Stokes-based solvers, the comparisons suggested that the athermal LBM should not be suitable for predictions of large fluctuations of density and temperature. Van Treek [12] presented the hybrid LBM to simulate a 3D turbulent heat transfer by using Smagorinsky sub-grid scale (SGS) model and validated laminar and turbulent natural convection in a cavity at various Rayleigh numbers up to $5 \times 10^{10}$ for Prandtl number $\operatorname{Pr}=0.71$ by means of relevant benchmark data. Sajjadi et al. [13] investigated the turbulent natural convection flow based on LBM coupled with LES. In this research, streamlines, local and average Nusselt numbers, and isotherm counters have been studied in different Rayleigh numbers. M. Fernandino et al. [14] carried out simulations of free surface duct flow with a flat interface with the LBM in combination with the Smagorinsky 
model, and the simulation results are in good qualitative agreement with the experiments. S. K. Kang et al. [15] adopted the Smagorinsky model for the turbulence simulation and investigated the effect of different 3D lattice models on the simulation results of wall-bounded turbulent flows in a circular pipe and in a square duct was investigated. M. Stiebler et al. [16] introduced a 3D lattice model with LES turbulence modeling on composite non-uniform grids, and studied the subcritical turbulent flows around a sphere in a channel are for a range of Reynolds numbers between 2000 and 10000, and the essential features of flows are captured. However, so far, an application of the flow in the pump intakes based on SRT (single-relaxation-time)-LBM model combined with LES has rarely been carried out. There is one case merely in using MRT (Multi-Relaxation-Time)-LBM code to simulate pump intake flow [17].

LBM with LES is capable to give more intrinsically predictions and explanations of swirling dominated flows from mesoscopic view. Besides, the LBM has some distinct advantages for simulating the flows in pump intake, such as, its high accuracy and the efficiency in the transient flows simulation, convenience to easily treat with complex geometry boundary conditions [18], simplicity of programming, intrinsic parallel algorithm, and so on [19-22]. Hence, SRT-LBM-LES model is adopted in predicting vortex flows inside open pump intakes in this paper, and compared with MRT-LBM model, the cost of computing decrease effectively under the same accuracy conditions. The numerical simulation based on the proposed SRT-LBM-LES combined model is validated by using a potently PIV data collected [23] on a scaled pump intake model consisting of one pump intake and one approaching channel. Moreover, this investigation extends the application scope of the SRT-LBM model.

The remaining parts of this paper are organized as follows. In Section 2, the numerical methods adopted in this study are described. The lattice Boltzmann equation and the LES turbulence model are given in this section. In Section 3, the model used in numerical simulations has the same size and configuration as the experimental model in relevant literature [23], and the initial and boundary conditions are consistent with the experiment. In Section 4, numerical calculations of the flow field in a pump intake for different operating conditions are carried out and the corresponding results are analyzed in details. The formation of the vortices (free-surface and wall-attached vortices) in the vicinity of the pump bell are discussed. Finally, in Section 5, the summary and conclusions of this study are provided.

\section{Lattice Boltzmann method}

Lattice Boltzmann equation (LBE) can be viewed as a special "discretization" form of continuous Boltzmann equation [24,25]. Eq. (2.1) is the evolution equation of the distribution function $f$ with discrete time for LBE

$$
f_{i}\left(x+e_{i} \Delta t, t+\Delta t\right)-f_{i}(x, t)=-\frac{1}{\tau}\left(f_{i}(x, t)-f_{i}^{(e q)}(x, t)\right),
$$


where $f_{i}$ is the distribution function for particles with velocity $e_{i}$ at position $x$ and time $t$ along the $i$ th direction of velocity, $\Delta t$ is the time step, $f_{i}^{(e q)}$ is the corresponding local equilibrium distribution, and $\tau$ is the single relaxation time.

The left-hand terms of the Eq. (2.1) model a streaming step for fluid particles while the right-hand terms express the collisions process through relaxation [26]

$$
\begin{array}{ll}
\text { collision: } & \tilde{f}_{i}(\boldsymbol{x}, t)=f_{i}(\boldsymbol{x}, t)-\frac{1}{\tau}\left(f_{i}(\boldsymbol{x}, t)-f_{i}^{(e q)}(\boldsymbol{x}, t)\right), \\
\text { streaming: } & f_{i}\left(\boldsymbol{x}+\boldsymbol{e}_{i} \Delta t, t+\Delta t\right)=\tilde{f}_{i}(\boldsymbol{x}, t),
\end{array}
$$

where $\tilde{f}_{i}$ is the post-collision distribution function.

A cubic lattice with 19 discrete velocity directions (D3Q19 model) adopted here is illustrated in Fig. 1.

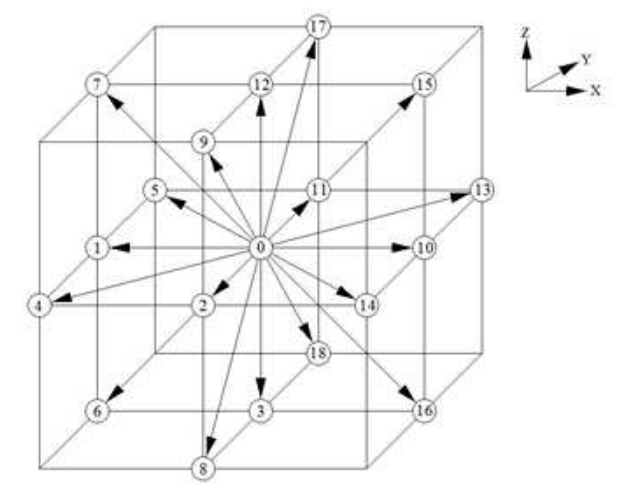

Figure 1: D3Q19 lattice model.

The particle speed velocity $\boldsymbol{e}_{\boldsymbol{i}}$ is defined as

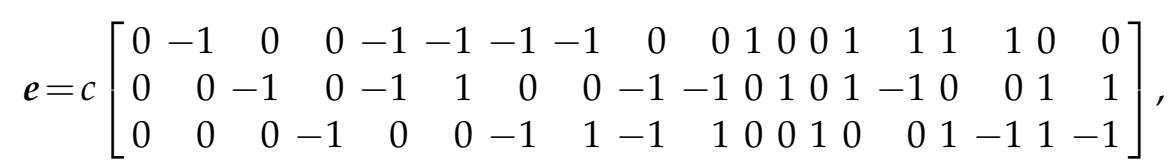

where $c=\Delta x / \Delta t, \Delta x$ is the lattice spacing.

In the present paper, D3Q19 lattice model is applied and the equilibrium distribution functions $f_{i}^{(e q)}$ is denoted as

$$
f_{i}^{(e q)}=w_{i} \rho\left[1+3 \boldsymbol{e}_{i} \cdot \boldsymbol{u}+\frac{9}{2}\left(\boldsymbol{e}_{\boldsymbol{i}} \cdot \boldsymbol{u}\right)^{2}-\frac{3}{2} \boldsymbol{u}^{2}\right] .
$$

The equilibrium distribution function $f_{i}^{(e q)}$ does not only depends on the local density $\rho$ but also macroscopic velocity $\boldsymbol{u}$. The weighting coefficient $w_{i}$ are given as follows

$$
w_{i}= \begin{cases}1 / 3 ; & i=0 \\ 1 / 18 ; & i=1, \cdots, 6 \\ 1 / 36 ; & i=7, \cdots, 18\end{cases}
$$


Once the distribution functions in the lattice Boltzmann equation are solved, the macroscopic variables, such as density $\rho$, macroscopic velocity $\boldsymbol{u}$ and pressure $p$ can be yielded by statistics from the first and two moments of the distribution functions by

$$
\rho=\int f d e=\sum_{i=0}^{18} f_{i}, \quad \boldsymbol{u}=\int e f d e=\frac{1}{\rho} \sum_{i=0}^{18} e_{i} f_{i}, \quad p=\frac{\rho}{3} c^{2}=\rho c_{s}^{2},
$$

where $c_{s}^{2}=c^{2} / 3$ is the sound speed of system.

In order to extend LBM applications and to well simulate turbulent flows of high Reynolds number, some turbulence models were combined with LBM. A statistical turbulence model $(k-\varepsilon)$ was introduced in LBM in Ref [27]. A. Pradhan [19] used the LBM to simulate turbulent flows in a channel using the sigma model for LES. K. N. Premnath [28] incorporated dynamic sub-grid scale (SGS) models in the LBM for LES of turbulent flows. Tang [29] coupled LBM and LES technology in the flow around a non-submerged groyne in a channel, without a significant change of the algorithm structure. In this paper, LES was implemented in LBM without a significant change of the algorithm, and based on eddy-viscosity assumptions, the Smagorinsky constant $\left(C_{S}\right)$ of the SGS model equal to 0.35. As shown in Ref. [29], the total relaxation time consists of the single relaxation time and the eddy relaxation time

$$
\tau_{e}=\tau+\tau_{s g s},
$$

where $\tau_{e}$ is the total relaxation time including the single relaxation time $\tau$ with respect to the molecular viscosity $v$ and the eddy relaxation time $\tau_{s g s}$ associated with turbulent eddy viscosity $v_{t}$, which determines the approaching rate to the local equilibrium.

The relation between the effective viscosity coefficient and the total relaxation time can be written as

$$
v_{e}=\frac{e^{2} \Delta t}{6}\left(2 \tau_{e}-1\right)
$$

In the meantime, the molecular viscosity coefficient and the single time is assumed to satisfy the relation

$$
v=\frac{e^{2} \Delta t}{6}(2 \tau-1)
$$

Then, the relationship between the eddy relaxation time and the turbulence viscosity can be obtained

$$
\tau_{s g s}=\frac{3 v_{t}}{e^{2} \Delta t}
$$

The turbulent eddy viscosity $v_{t}$ is given by

$$
v_{t}=\left(C_{s} \bar{\Delta}\right)^{2} \sqrt{2 \bar{S}_{i j} \bar{S}_{i j}}
$$

where the strain-rate tensor $\bar{S}_{i j}$ is related to the non-equilibrium momentum flux tensor by using the Chapman-Enskog expansion. It is needs to be stressed that the LBM has 
ability to calculate $\bar{S}_{i j}$ in terms of the distribution function

$$
\bar{S}_{i j}=-\frac{3}{2 e^{2} \rho\left(\tau+\tau_{s g s}\right) \Delta t} \sum_{k} e_{k i} e_{k j}\left(f_{k}-f_{k}^{e q}\right) .
$$

Combining Eqs. (2.11) and (2.12), the eddy relaxation time is expressed as

$$
\tau_{s g s}=\frac{3}{e^{2} \Delta t} v_{t}=\frac{3}{e^{2} \Delta t}\left(C_{s} \bar{\Delta}\right)^{2} \sqrt{2 \bar{S}_{i j} \bar{S}_{i j}}
$$

thus, the eddy relaxation time is

$$
\tau_{s g s}=-\frac{9\left(C_{s} \bar{\Delta}\right)^{2}}{2 e^{4} \rho\left(\tau+\tau_{s g s}\right) \Delta t^{2}} \sqrt{2 \Pi_{i j} \Pi_{i j}}
$$

where $\Pi_{i j}=\sum_{k} e_{k i} e_{k j}\left(f_{k}-f_{k}^{e q}\right)$.

In the equally spaced rectangular grids, $\bar{\Delta}=\Delta x=\Delta y=\Delta z$, Eq. (2.14) can further be written as

$$
\tau_{s g s}=\frac{9 C s^{2}}{2 e^{2} \rho\left(\tau+\tau_{s g s}\right)} \sqrt{2 \Pi_{i j} \Pi_{i j}},
$$

where the relaxation time of SGS model is

$$
\tau_{\text {sgs }}=\frac{-\tau+\sqrt{\tau^{2}+18\left(C_{s} \bar{\Delta}\right)^{2} \sqrt{2 \Pi_{i j} \Pi_{i j}} /\left(e^{4} \rho \Delta t^{2}\right)}}{2} .
$$

The total relaxation time is defined as

$$
\tau_{e}=\tau+\tau_{s g s}=\frac{\tau+\sqrt{\tau^{2}+18\left(C_{s} \bar{\Delta}\right)^{2} \sqrt{2 \Pi_{i j} \Pi_{i j}} /\left(e^{4} \rho \Delta t^{2}\right)}}{2} .
$$

Finally, the equivalent relaxation time of LBM-LES combination model can be given by coupling Smagorinsky model with LB equation

$$
f_{i}\left(x+e_{i} \Delta t, t+\Delta t\right)-f_{i}(x, t)=-\frac{1}{\tau_{e}}\left(f_{i}(x, t)-f_{i}^{(e q)}(x, t)\right) .
$$

\section{Numerical investigations}

The numerical investigations were carried out with the LBM code. For turbulence model, a Smagorinsky SGS model was applied, and SRT model was embodied in collision models. 


\subsection{Pump intake geometry}

To validate the numerical solutions, the geometrical characteristics of the pump intake in this study are the same as those of the single-pump intake model used in the laboratory experiments [23], which is a representative research of the free surface intake. The geometry of the calculated single pump intake is exhibited in Fig. 2. The structure comprises a single pump bell and a long rectangular channel with free surface.

The interior diameter $D=100 \mathrm{~mm}$ of the pipe is treated as the characteristic length scale. The width of the channel is $L y=3.0 \mathrm{D}$ and the height of the computation domain, $H$ is 3.0D. $H_{f}$ is the submergence water depth and the $H_{p}=D$ is the height of the pump bell from the floor. In order to guarantee the results not to be affected by the inlet conditions and the flows far away from the bell to be fully developed ones, the length $L_{x}$ of the channel is $6 D$, and similarly, to ensure that the flows at the outlet are fully-developed one [30], the height is $H=3.0 D, L_{x}$ and $H$ are the same as those in literature. The back wall of the pump intake $L_{b}$ is situated $1.1 D$ distant from the center of the pump bell in the downstream direction and the inlet sections are situated $4.9 \mathrm{D}$ distant form the bell center in the upstream direction. The center of the pump bell is placed dissymmetry between the two side walls, and there is an extremely small deviation distance of $3.3 \%$ of the channel width from bell center in the $y$ direction [23]. The distance from the pipe bell center to the side wall 1 (L1) is equal to $0.65 D$, and the other distance from the pipe bell
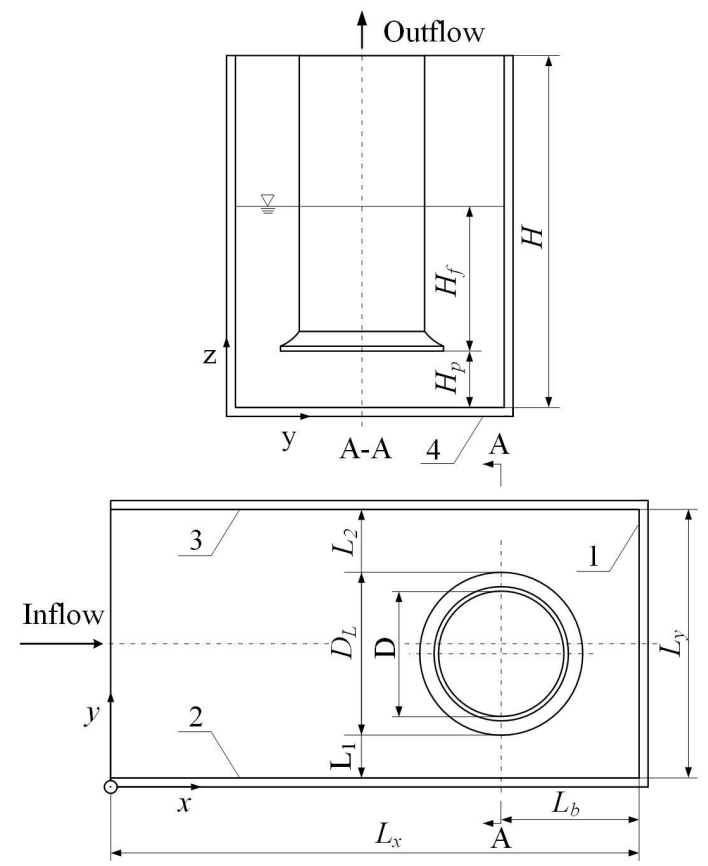

1. back wall, 2 . side wall 1,3 . side wall 2 , 4 . floor

Figure 2: Pump intake geometry and main parameters. 
Table 1: Pump intake geometrical parameters.

\begin{tabular}{||ccc||}
\hline$L_{y} / D=3.0$ & $H / D=3.0$ & $L_{x} / D=6.0$ \\
\hline$L_{b} / D=1.1$ & $L_{1} / D=0.65$ & $L_{2} / D=0.85$ \\
\hline
\end{tabular}

Table 2: Operating conditions.

\begin{tabular}{||ccc||}
\hline Cases & Flow rate $Q\left(\mathrm{~m}^{3} / \mathrm{min}\right)$ & Submergence $\left(H_{f} / D\right)$ \\
\hline Case1 & 1.0 & 1.3 \\
Case2 & 0.6 & 1.3 \\
Case3 & 1.0 & 0.5 \\
\hline
\end{tabular}

Table 3: Pump intake physical parameters.

\begin{tabular}{||cccc||}
\hline Cases & Re & We & Fr \\
\hline Case1 & $2.122 \times 10^{5}$ & 2800 & 1.02 \\
Case2 & $1.273 \times 10^{5}$ & 1521 & 0.76 \\
Case3 & $2.212 \times 10^{5}$ & 3597 & 1.16 \\
\hline
\end{tabular}

center to the side wall $2 L_{2}=0.85 D$. The diameter of the pump bell $D_{L}$ is $1.5 D$. All the parameters are listed in Table 1.

Three different conditions with different flow rate $Q$ and submergence water depth $H_{f}$ of the pump bell listed in Table 2 are considered. The Reynolds numbers inside the intake pipe are $2.122 \times 10^{5}, 1.273 \times 10^{5}$, and $2.122 \times 10^{5}$ in three cases respectively. The corresponding Weber number is high enough for surface tension effects to be negligible. The Froude number is the major controlling parameter [31], which is low enough to avoid air entraining vortices and to justify the assumption of a flat free surface [1]. The detailed pump intake physical parameters are listed in Table 3, and the parameters of flowing medium (water at $20^{\circ} \mathrm{C}$ ) are $\rho=1000 \mathrm{~kg} / \mathrm{m}^{3}, \sigma=0.072 \mathrm{~N} / \mathrm{m}, v=1 \times 10^{-6} \mathrm{~m}^{2} / \mathrm{s}, g=9.81 \mathrm{~m} / \mathrm{s}^{2}$, respectively.

\subsection{Computational mesh}

The quality of the computational mesh is important in achieving the desired accuracy of the simulations. The grid independent analyses were carried out, and finally an adopted block-structured mesh with 432000 elements is displayed in Fig. 3. The single-pump intake computation domain has $121 \times 61 \times 61$ grid points in the $x, y$, and $z$ directions, respectively.

\subsection{Initial and boundary conditions}

As mentioned previously, the Weber number in each case is high enough to neglect the effect of surface tension [32], and the Froude number is low enough to guarantee the 


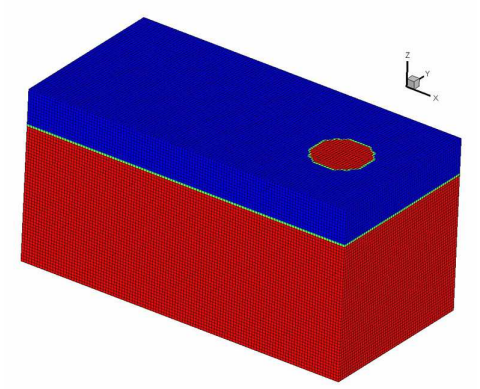

(a) 3D Grid

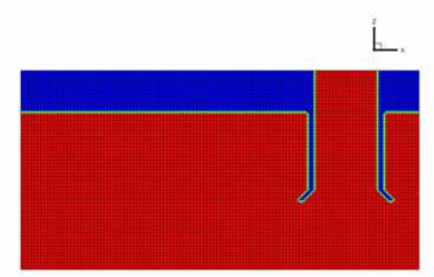

(b) X-Z plane $(y=28)$

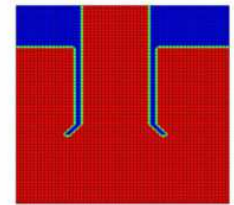

(c) Y-Z plain $(x=98)$

Figure 3: Grid scheme of the computation domain.

ratio of the force of gravity and the inertia force have to be identical for the numerical model and the prototype structure. In the experiments [23] it is known that the surface was almost plat. Hence, the three operating cases in this pump intake were calculated as a single-phase simulation with a free surface, and rigid assumption is adopted to treat with the free surface during the simulations, which is a typical treatment approach in single-phase simulations of pump intake models and used by S. G. Constantinescu et al. [32], V. P. Rajendran et al. [33], and Tomoyoshi Okamura et al. [23].

The velocity components $u=u_{0}$ and $w=v=0$ were specified at the inlet nodes, and $u_{0}$ is calculated by the flow rate in each case. The pressure boundary condition was specified at outlet and the condition of zero gradient was implemented to the velocity in $z$ direction to meet global mass conservation. The velocity of outlet is corrected according to the flow rate of the inlet. At all other nodes the velocities are set as $u=v=w=0$. The initial densities in flow field are taken as $1000 \mathrm{~kg} / \mathrm{m}^{3}$. The no-slip condition was applied for the velocity components at all solid walls. It is noted that the free surface is treated as a flat plane and as a free slip wall without friction. Numerically, the reflection boundary condition is imposed on the lattice-orientated flat plane to guarantee a free slip condition on the free surface. In microscopic, the initial distribution functions on all lattice points are the equilibrium distribution functions. The particle velocity $c=1$. The lattice spacing is $0.005 \mathrm{~m}$ and the time step $\Delta t=2 \times 10^{-6} \mathrm{~s}$.

\section{Computational results and analyses}

The experimental data in the intake [23] were used to validate the above-mentioned model and numerical algorithms, the laser light sheet method was used to obtain locations of the free surface vortices and the PIV was employed to obtain the velocity distributions at the bell entrance. The configuration dimensions and the flow parameters used in the simulation are identical to those in the literature. 


\subsection{Comparisons of free surface vortex cores}

In order to carry out the quantitative analyses on the vorticity of the vortex vector on the free surface vortices, the vortex vector is calculated by using the expression (4.1), and the $z$-component $\omega_{z}$ of the vortex vector converted to the corresponding dimensionless values $\tilde{\omega}_{z}$ by using the expression (4.2)

$$
\begin{aligned}
& \boldsymbol{\omega}=\frac{1}{2} \nabla \times \boldsymbol{u} \\
& \tilde{\omega}_{z}=\left|\omega_{z}\right| / \omega_{z-r e f}
\end{aligned}
$$

where $\omega_{z-r e f}$ is the average $z$-component vorticity as a reference value, which computed by using the expression (4.3), and its value is $9.981 / \mathrm{s}$

$$
\omega_{z-r e f}=\frac{1}{n} \sum_{1}^{n}\left|\omega_{z}\right|
$$

Fig. 4 reveals a good agreement between the simulations (drawn as squares) and experimental data (drawn as solid circles) concerning the positions of the free surface vortex cores for case 1 . The relative coordinates are expressed in dimensionless quantities. The region of experimental data is marked by a dot circle with center coordinates $(0.40,0.94)$ and radius 0.44 . The predicted position coordinates of the free surface vortex cores are $(0.68,0.84),(0.49,1.02),(0.29,1.02),(0.74,1.98)$, respectively, which are labelled by the digits $1,2,3$ and 4 . To be specific, the positions of two dominant free surface vortices labelled by the digits $2\left(\tilde{\omega}_{z}=1.72\right)$ and $3\left(\tilde{\omega}_{z}=0.65\right)$ between the pump bell and side wall 1 locate in this circle, and the other vortex labelled by the digits $1\left(\tilde{\omega}_{z}=1.28\right)$ is close to it. Meanwhile, another small free surface vortex (labelled by the digits 4 ) between the pump bell and side wall 2 is also numerically captured. Compared to the dominant vortices, this

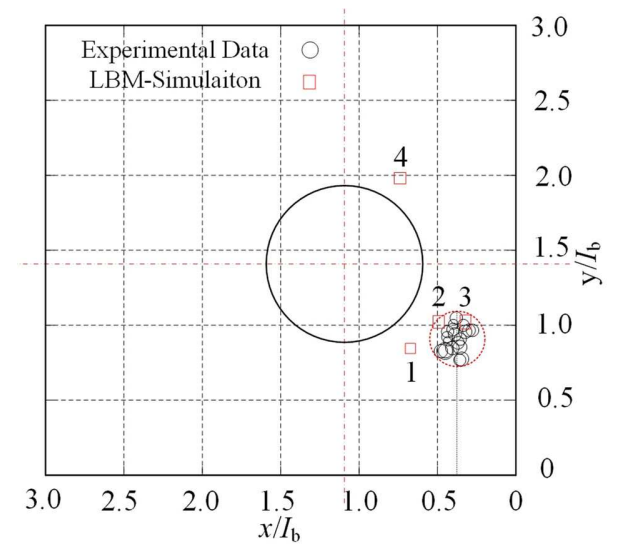

Figure 4: Comparisons of the location of free surface vortex for Case 1. 


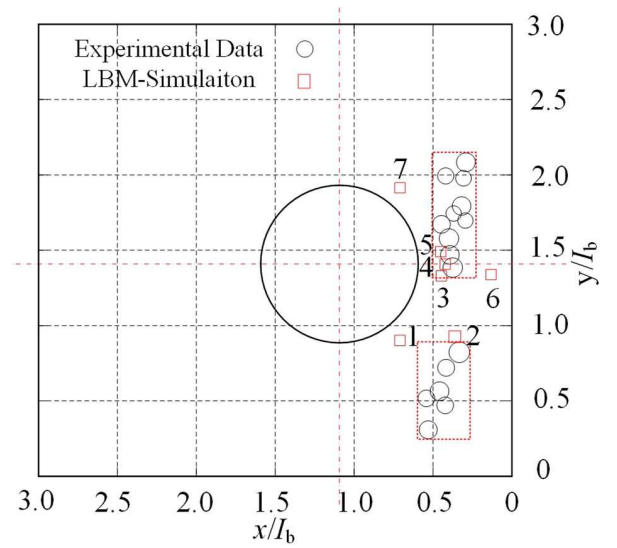

Figure 5: Comparisons of the location of free surface vortex for Case 3.

small vortex vorticity is too weak $\left(\tilde{\omega}_{z}=0.35\right)$, which cannot easily be detected by experiment.

The number and position of the free surface vortices for case 3 is drawn in Fig. 5 to compare with the experimental data. The circles represent the experimental positions of the real vortex cores, and the squares stand for the numerical simulation ones. The two regions of experimental data concerning vortex range are drawn as red dot rectangle respectively, and distributed on the side of the pump bell, which locate in the range of $x$-axis interval of $[0.25,0.51]$ and $y$-axis interval of $[1.31,2.15]$, and in the other range of $x$-axis interval of $[0.24,0.60]$ and $y$-axis interval of $[0.25,0.89]$. The predicted position coordinates of the free surface vortex cores are $(0.71,0.90),(0.36,0.92),(0.44,1.32),(0.43,1.42)$, $(0.46,1.49),(0.13,1.34),(0.72,1.92)$, respectively, which are labelled by the digits 1 to 7 . There are predicted five different vortices (labelled by the digits 2 to 6 ) between the pump bell and back wall, which are almost located in the corresponding experimental regions. Besides, the other two small vortices (digits 1 and 7) approximately symmetrically distributed on the side of the pump bell are captured. All the comparisons showed that the predicted results match well with the measured.

\subsection{Analyses of vortex cores and streamlines}

To better describe the vertex structure in the intakes, the time-averaged streamlines in various planes are presented in Fig. 6. The "free surface" plane is defined as the plane under the corresponding free surface by $0.05 \times H_{f}$, and the "floor" plane is $0.3 D$ above the floor boundary. The plane signed "side wall 1" is situated $0.4 L_{1}$ and "side wall 2" is away $0.13 L_{2}$ from the according physical boundary. The "back wall" plane is located $0.25 L_{b}$ from the corresponding back wall.

A small floor attached vortex under the pump bell, two small back-wall vortices, sidewall vortices and free-surface vortices are shown in Fig. 6.

For a more vivid and stereo description of these predicted vortex structures, the time- 


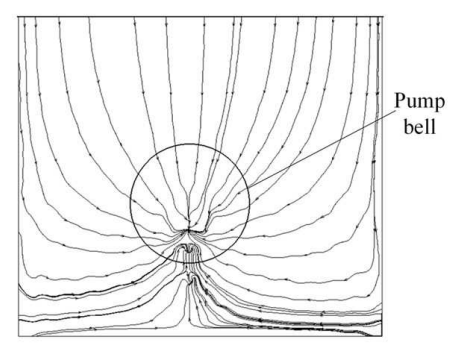

(a) floor

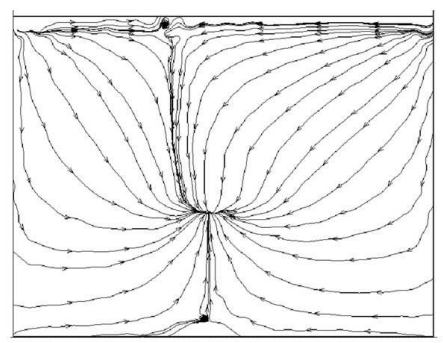

(b) back wall

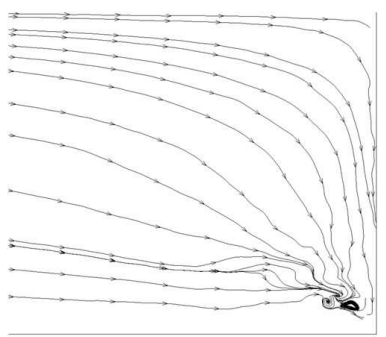

(c) side wall 1

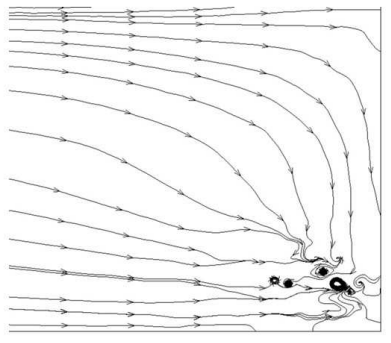

(d) side wall 2

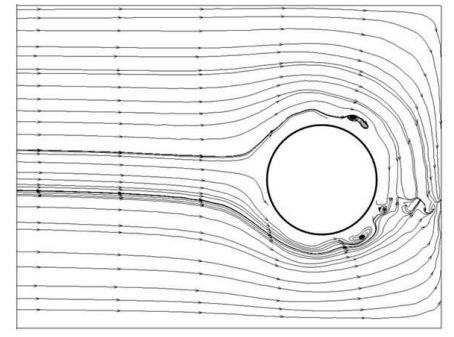

(e) free surface

Figure 6: Surface streamlines for Case 1.

dependent evolutions of the free surface vortices for case 1 are shown in Fig. 7(a) and (b) from $t=16.8 \times 10^{4}$ time steps to $t=17.0 \times 10^{4}$ time steps and the time-dependent evolutions of the wall-attached vortices for case 1 are illustrated by means of streamlines in Fig. 7(c) and (d). Fig. 7(a) displays that one small vortex formed near the vertical plane when $y=0.014 m$ between pump bell and side wall 1 at $t=16.8 \times 10^{4}$ time steps. After 2000 time steps later, i.e.t $=17.0 \times 10^{4}$ time steps, this vortex become strong, and the accompanying swirling flow near the vertical plane when $y=0.014 \mathrm{~m}$ is shown in Fig. 7(b). Besides, the numerical results show that this free surface vortex is intermittent, in another more 2000 time steps later, there is no obvious free surface vortex exist and the corresponding picture is not given. Fig. 7(c) shows the wall-attached vortex at $t=16.8 \times 10^{4}$ time steps, there are two floor-wall-attached vortices are formed in floor near the back wall, and a quite small back-wall-attached vortex locates in the middle-lower position of the back wall. After 2000 time steps later, in Fig. 7(d), a bigger back-wall-attached vortex locates in the middle-lower position of the back wall, and more floor-wall-attached vortices are formed in floor near the back wall. Fig. 7(e) and (f) shows the wall-attached vortex at $t=$ $17.2 \times 10^{4}$ time steps when $x=0.49 \mathrm{~m}$. Fig. $7(\mathrm{e})$ displays that one floor-wall-attached vortex reappears in floor under the pump bell. At the same time, Fig. 7(f) demonstrates one wall-attached vortex and its amplified structure in right-bottom corner. These different snapshots reveal the changes of the location and the structure of vertices with time.

As the submergence water depth decreases, various free-surface vortices reproduce 


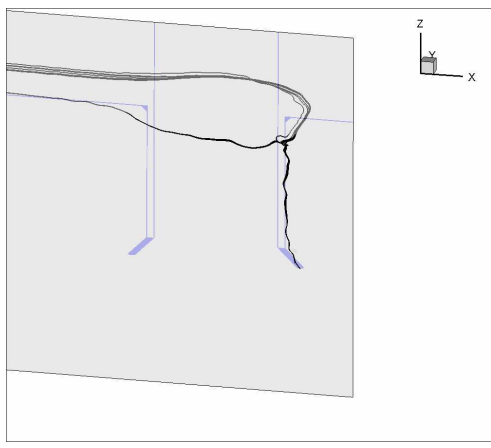

(a) $t=16.8 \times 10^{4}$ time steps

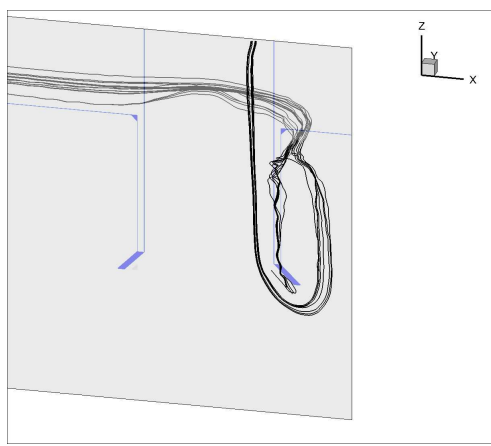

(b) $t=17.0 \times 10^{4}$ time steps

(a) \& (b) free surface vortices

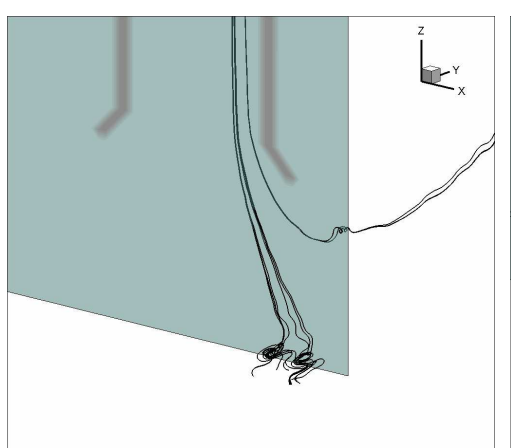

(c) $t=16.8 \times 10^{4}$ time steps

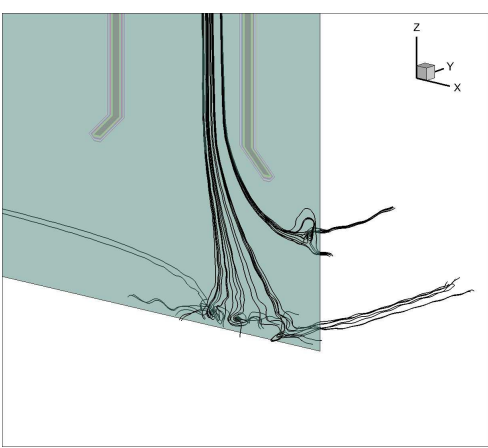

(d) $t=17.0 \times 10^{4}$ time steps

(c) \& (d) wall-attached vortices when $y=0.014 \mathrm{~m}$

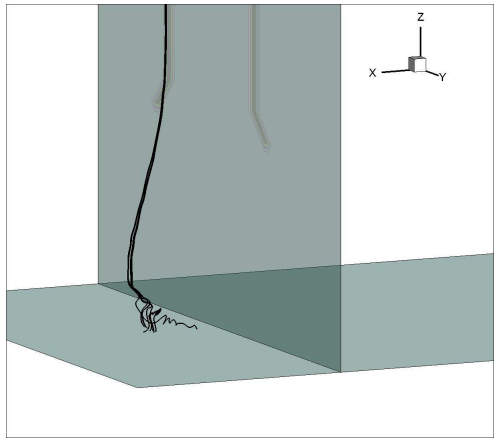

(e) $t=17.2 \times 10^{4}$ time steps

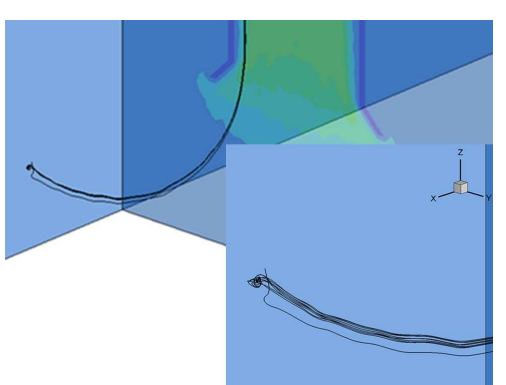

(f) $t=17.2 \times 10^{4}$ time steps

(e) \& (f) wall-attached vortices when $x=0.49 \mathrm{~m}$

Figure 7: Free surface and wall-attached vortices for Case 1.

quickly and are instable compared to those for case 1 because higher water depth can suppress the free-surface vortices to form. Fig. 8 shows the free-surface streamlines for case 3 at three different moments in the same free surface plane. Fig. 8(a), shows that two 


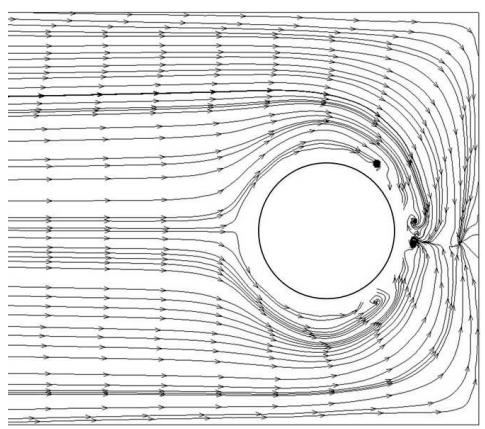

(a) $t=17.0 \times 10^{4}$ time steps

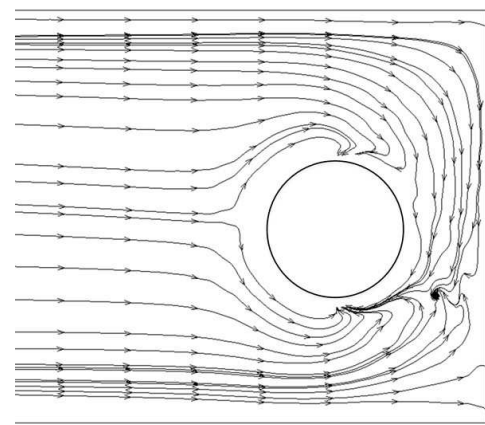

(b) $t=17.2 \times 10^{4}$ time steps

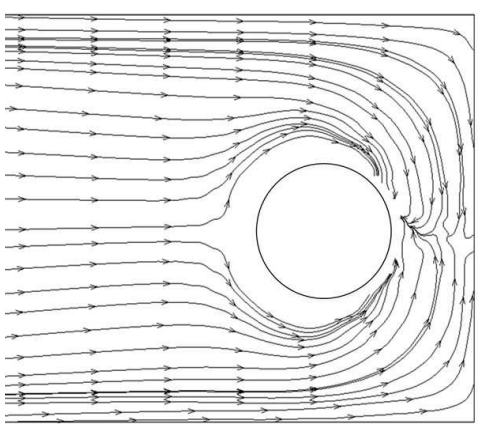

(c) $t=17.4 \times 10^{4}$ time steps

Figure 8: Free-surface streamlines for Case 3 at different time.

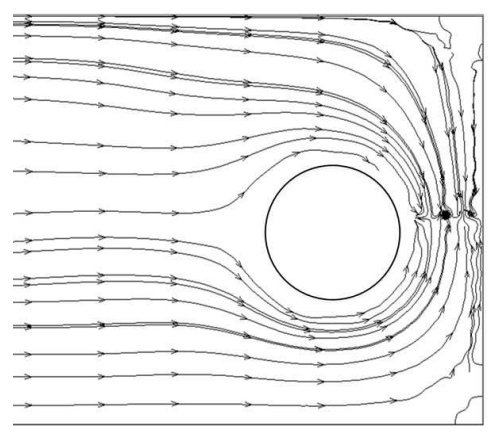

Figure 9: Surface streamlines for Case 2.

vortices approximately symmetry distributed on the side of the pump bell at moment 1 , and moreover, four other vortices between the pump bell and back wall are observed. Only one free-surface vortex between the pump bell and side wall 1 shown in Fig. 8(b) at the moment 2 , and the corresponding surface streamlines are different from those at moment 1 . However, there is no existing vortex at the moment 3 shown in Fig. 8(c).

The submergence depth for case 2 is the same as case 1 , but the flow rate is 0.6 times of that for case 1 , so the flow conditions for case 2 are below the critical flow ones, and then its flow and vortices are smooth and stable. Fig. 9 illustrated the surface streamlines for case 2 at a certain moment in the same free surface plane, and two obvious vortices between the pump bell and back wall are observed.

\subsection{Velocity comparisons}

The PIV measurements of Ref. [23] are used to carry out a quantitative analysis of the calculations for case 1 . These measurements are available along a measured line intersected by the plane located $0.85 \mathrm{D}$ above the floor and the plane $1.1 \mathrm{D}$ distant from the back wall as shown in Fig. 10. 


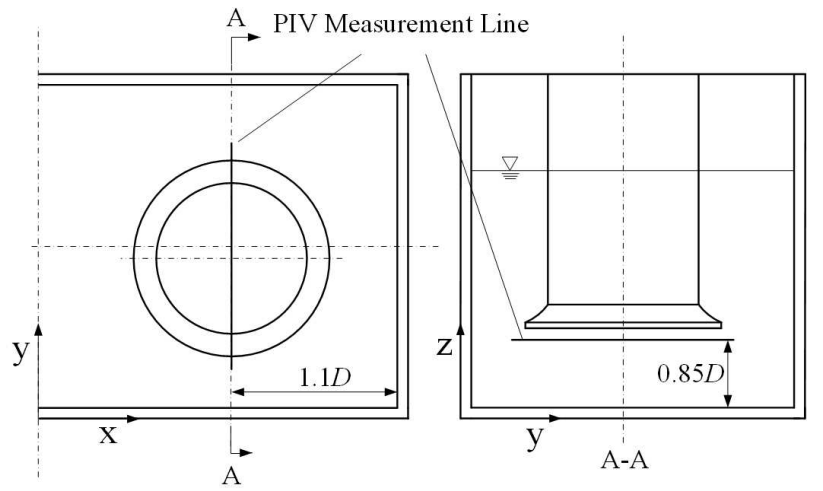

Figure 10: PIV measurement line.

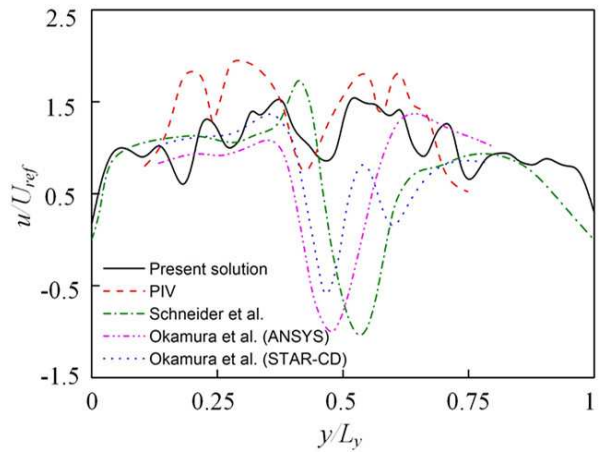

(a)

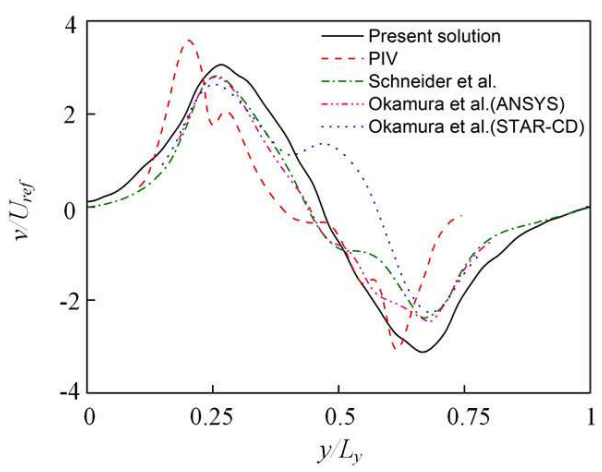

(b)

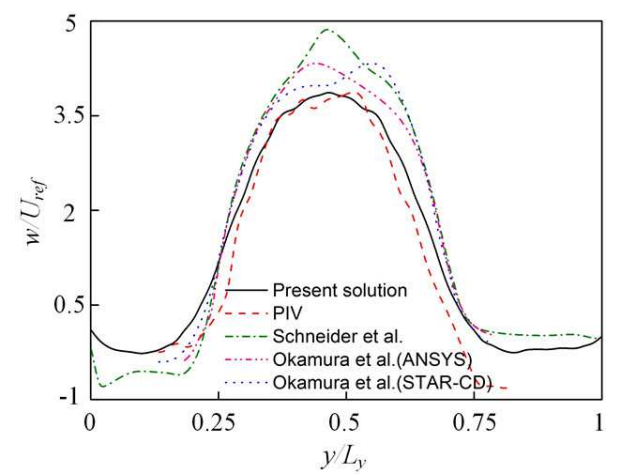

(c)

Figure 11: Comparison of LBM with experiment for Case 1.

Fig. 11 displays comparisons of the velocity distributions along the measured line between the PIV measurements and the numerical results where the velocity components are converted dimensionless values by means of the average velocity in the intake 


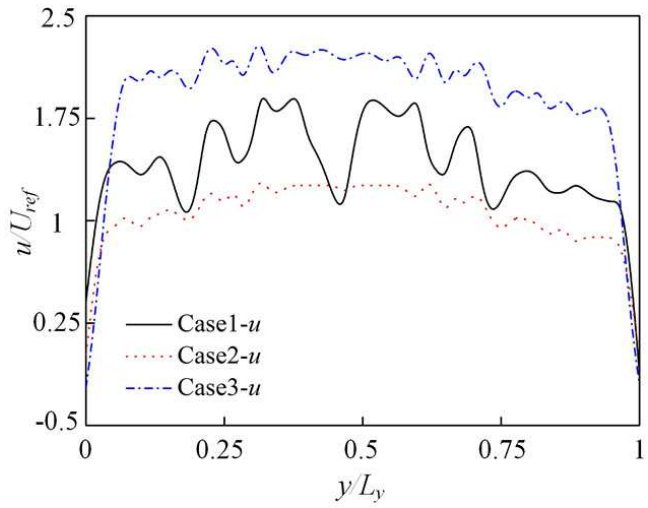

(a)

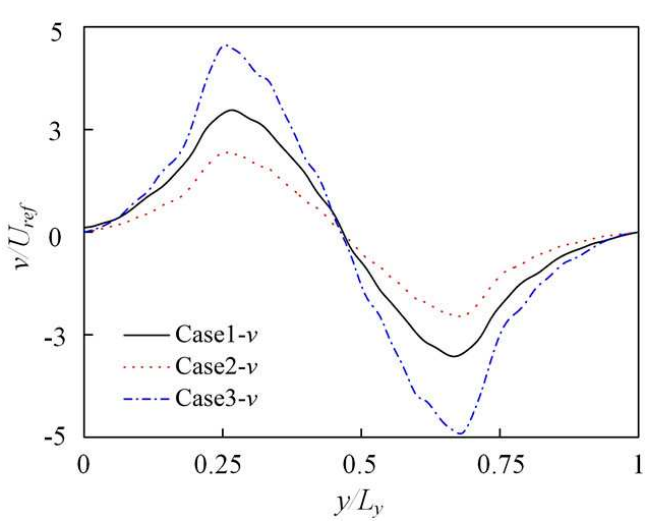

(b)

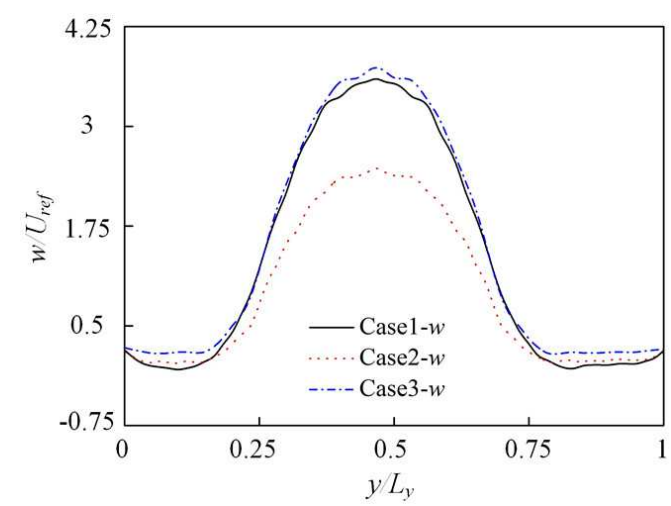

(c)

Figure 12: Comparison of LBM with experiment for all working points.

channel $U_{r e f}$, and $u, v, w$ stand for the velocity components in the $x, y, z$ directions, respectively. All the numerical results have same tendencies with the experimental, but compared to these numerical results from Ref. [10] and Ref. [23], the velocity distribution predicted by this present model is closer to the PIV data, especially, $x$-velocity distributions shown in Fig. 11(a).

Fig. 12 shows the calculated velocity profiles at three cases along the PIV measurement line. The $x$-velocity and the related-near-wall velocity gradient increases as the flow rate increase, and their tendency of $x$-velocity profiles for these three cases are similar, as shown in Fig. 12(a). The fluctuation of $x$-velocity for CWL is higher than those for case 2 and 3. The velocity profiles of the $y$-component (Fig. 12(b)) are similar for all cases. The velocity profiles of the z-component (Fig. 12(c)) are similar for all cases, but the peaks of the $w$-velocity curves for case 2 is lower than those for case 1 and 3 which are close to each other, and these are due to the different vortex structures under the pump bell, different values of $H_{f}$ and the different flow rates. 


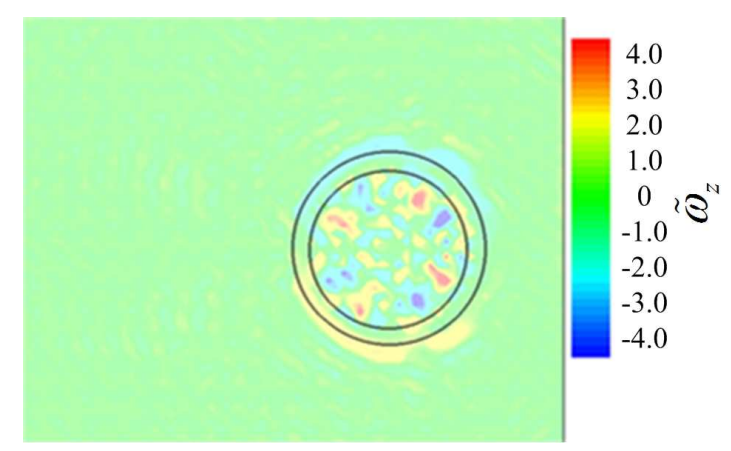

Figure 13: Vorticity distribution of LBM for Case 1 at $z=44$.

Fig. 13 shows the $z$-component distribution of the vorticity vector at the bell entrance for case 1 . The $z$-component $\omega_{z}$ of the vorticity vector are converted to the corresponding dimensionless values by using Eqs. (4.1)-(4.3), the reference value of vorticity $\omega_{z-r e f}$ is set to the value of $9.981 / \mathrm{s}$ on the free surface. The vorticity distribution of LBM is qualitatively similar in strength to the results in the literature [23].

\section{Conclusion}

SRT-LBM model combined with LES is proposed to predict various vortex structures and their evolutions in open pump intakes.

For the various free-surface vortex structures for CWL, two dominant ones between the pump bell and side wall 1, and a small one between the pump bell and side wall 2 are predicted. Besides, a number of wall-attached vortices in the side walls, back wall and floor are predicted. As the submergence water depth decreases, six free-surface vortices (including approximately symmetric on each side of the pump bell and four other ones between the pump bell and back wall) reproduce and diminish randomly. These predicted free-surface vortices for case 3 are instable compared to those for CWL. For case 2, the flow and vortices are smooth and stable. The predicted vortex locations are in good agreement with the experimental data.

At the bell entrance, the velocity distributions along the bell diameter in three directions obtained in this paper have same tendencies with the experimental, and the velocity profiles are similar for all cases.

As previously mentioned, the numerical model is used to understand the complex flow phenomena existed in realistic pump intake better. From mesoscopic view, the LBMLES as a predictive tool can be widely applied in the design or redesign process of pump intakes. Furthermore, LBM-LES model combined with VOF method can be treated as an alternative potential approach for predicting free-surface vortex and deserve further exploration in the future. 


\section{Acknowledgments}

This work was supported by the National Natural Science Foundation of China (Grant Nos. 51479196, 51179192, 51139007), the Program for New Century Excellent Talents in University (NCET) (Grant No. NETC-10-0784).

\section{References}

[1] M. Padmanabhan and G. E. Hecker, Scale Effects in Pump Sump Models, Journal of Hydraulic Engineering, 1984, 110 (11), 1540-1556.

[2] L. Cristofano and M. Nobilia, Influence of boundary conditions in numerical simulation of free surface vortices, Energy Procedia, 2015, (82), 893-899.

[3] N. Yildirim and F. Kocabas, Critical Submergence for Intakes in Open Channel Flow, Journal of Hydraulic Engineering, 1995, 121(12),900-905.

[4] Y. Zhang and $\mathrm{Y}$. Wu, A review of rotating stall in reversible pump turbine, Proceedings of the Institution of Mechanical Engineers, Part C: Journal of Mechanical Engineering Science, 2017, (4), 1181-1204.

[5] V. P. Rajendran and V. C. Patel, Measurement of vortices in a model pump-intake bay by PIV, Journal of hydraulic Engineering, 2000, 126 (5), 322-334.

[6] J. Matsui, K. Kamemoto and Okamura, et al., CFD benchmarks and a model experiment on the flow in pump sump, Proc. of 23rd IAHR Symposium Yokohama, (2006).

[7] T. E. Tokyay and S. G. Constantinescu, Validation of a Large-Eddy Simulation Model to Simulate Flow in Pump Intakes of Realistic Geometry, Journal of hydraulic Engineering, 2006, 132(12), 1303-1315.

[8] X. L. Tang, F. J. Wang and Y. J. Li et al., Numerical investigations of vortex flows and vortex suppression schemes in a large pumping-station, Mechanical Engineering Science, Proc. IMechE, 2010, 225, 1459-1480.

[9] A. Skerlavaj, L. Skerget and J. Ravnik, et al., Predicting Free-Surface Vortices with SinglePhase Simulations, Engineering Applications of Computational Fluid Mechanics, 2014, 8(2), 193-210.

[10] X. L. Tang, W. C. Wang and F. J. Wang, et al., Application of LBM-SGS Model to Flows in a Pumping-Station Forebay, Journal of Hydrodynamics, 2010, 22(2), 196-206.

[11] D. Bespalko, A. Pollard and M. Uddin, Analysis of the pressure fluctuations from an LBM simulation of turbulent channel flow, Computers \& Fluids, 2012, 54(2), 143-146.

[12] C. V. Treeck, E. Rank and M. Krafczyk, et al., Extension of a hybrid thermal LBE scheme for large-eddy simulations of turbulent convective flows, Computers \& Fluids, 2006, 35(8), 863-871.

[13] H. Sajjadi, M. Gorji and S. Hosseinizadeh, et al., Numerical Analysis of Turbulent Natural Convection in Square Cavity using Large-Eddy Simulation in Lattice Boltzmann Method, Iranian Journal of Science \& Technology Transactions of Mechanical Engineering, Trans. BEng., 2012, 35 (M2), 133-142.

[14] M. Fernandino, K. Beronov and T. Ytrehus, Large eddy simulation of turbulent open duct flow using a lattice Boltzmann approach, Mathematics \& Computers in Simulation, 2009, 79(5), 1520-1526.

[15] S. K. Kang and Y. A. Hassan, The effect of lattice models within the lattice Boltzmann method in the simulation of wall-bounded turbulent flows, Journal of Computational Physics, 2013, 
232(1), 100-117.

[16] M. Stiebler, M. Krafczyk and S. Freudiger, et al., Lattice Boltzmann large eddy simulation of subcritical flows around a sphere on non-uniform grids, Computers \& Mathematics with Applications, 2011, 61(12), 3475-3484.

[17] A. Schneider, D. Conrad and M. Bohle, Lattice Boltzmann Simulation of the Flow Field in Pump Intakes-A New Approach, Journal of Fluids Engineering, 137 (2015) 1-10.

[18] S. K. Bhaumik and K. N. Lakshmisha, Lattice Boltzmann simulation of lid-driven swirling flow in confined cylindrical cavity, Computers \& Fluids, 2007, 36(7), 1163-1173.

[19] A. Pradhan and S. Yadav, Large Eddy Simulation using Lattice Boltzmann Method based on Sigma Model, Procedia Engineering, 2015, 127, 177-184.

[20] S. Succi, V. I. Karlin and H. Chen, Role of the H theorem in lattice Boltzmann hydrodynamic simulations, Review of Modern Physics, 2002, 74(4),1203-1220.

[21] A. J. C. Ladd and R. Verberg, Lattice-Boltzmann Simulations of Particle-Fluid Suspensions, Journal of Statistical Physics, 2001, 104(5), 1191-1251.

[22] D. Yu, R. Mei and L. S. Luo, et al., Viscous flow computations with the method of lattice Boltzmann equation, Progress in Aerospace Sciences, 2003, 39(5), 329-367.

[23] Tomoyoshi Okamura, Kyoji Kamemoto, and Jun Matsui, CFD Prediction and Model Experiment on Suction Vortices in Pump Sump, The 9th Asian International Conference on Fluid Machinery, 2007, 10.

[24] X. Y. He and L. S. Luo, Theory of the lattice Boltzmann method: From the Boltzmann equation to the lattice Boltzmann equation, Physical Review E, 1997, (56), 6811-6817.

[25] X. Y. He and L. S. Luo. A priori derivation of the lattice Boltzmann equation, Physical Review E, 1997, (55), 6333-6336.

[26] R. Mei, Li-Luo S. and Shyy W, An accurate curved boundary treatment in the lattice Boltzmann method, Institute for Computer Applications in Science and Engineering (ICASE), 2006.

[27] C. M. Teixeira, Incorporating Turbulence Models into the lattice-Boltzmann method, International Journal of Modern Physics C, 1998, 9(8), 1159-1175.

[28] K. N. Premnath, M. J. Pattison and S. Banerjee, Dynamic sub-grid scale modeling of turbulent flows using lattice-Boltzmann method, Physica A Statistical Mechanics \& Its Applications, 2009, 388(13), 2640-2658.

[29] X. Yu, X. L. Tang and W. C. Wang, et al., A lattice Boltzmann model coupled with a Large Eddy Simulation model for flows around a groyne, International Journal of Sediment Research, 2010, 25(3), 271-282.

[30] E. J. Hemingway, P. Mishra and M. C. Marchetti, et al., Correlation lengths in hydrodynamic models of active nematics, Soft Matter, 2016, 12(38), 7943.

[31] J. A. Odgaard, Free-Surface Air Core Vortex, Journal of Hydraulic Engineering, 1986, 112, 610-620.

[32] S. G. Constantinescu and V. C. Patel, Numerical Model for Simulation of Pump-Intake Flow and Vortices, Journal of Hydraulic Engineering, 1998, 124(2), 123-134.

[33] V. P. Rajendran, S. G. Constantinescu and V. C. Patel, Experimental validation of numerical model of flow in pump-intake bays, Journal of Hydraulic Engineering, 1999, 125(11), 11191125 . 